Accepted to the ASTRONOMICAL JOURNAL 26 November 2003

Preprint typeset using $\mathrm{LAT}_{\mathrm{E} X}$ style emulateapj v. 04/03/99

\title{
MASSIVE FIELD STARS AND THE STELLAR CLUSTERING LAW
}

\author{
M. S. OEY AND N. L. KING \\ Lowell Observatory, 1400 W. Mars Hill Rd., Flagstaff, AZ 86001; Sally.Oey@lowell.edu \\ AND \\ J. WM. PARKER \\ Department of Space Studies, Southwest Research Institute, Suite 426, 1050 Walnut St., Boulder, CO 80302
}

Accepted to the ASTRONOMICAL JOURNAL 26 November 2003

\begin{abstract}
The distribution of $N_{*}$, the number of OB stars per association or cluster, appears to follow a universal power-law form $N_{*}^{-2}$ in the local Universe. We evaluate the distribution of $N_{*}$ in the Small Magellanic Cloud using recent broadband optical and space-ultraviolet data, with special attention to the lowest values of $N_{*}$. We find that the power-law distribution in $N_{*}$ continues smoothly down to $N_{*}=1$. This strongly suggests that the formation of field massive stars is a continuous process with those in associations, and that the field stars do not originate from a different star formation mode. Our results are consistent with the model that field massive stars represent the most massive members in groups of smaller stars, as expected if the clustering law applies to much lower masses as is expected from the stellar initial mass function (IMF). These results are consistent with the simultaneous existence of a universal IMF and a universal clustering law. Jointly, these laws imply that the fraction of field OB stars typically ranges from about $35 \%$ to $7 \%$ for most astrophysical situations, with an inverse logarithmic dependence on the most populous cluster, and hence, on galaxy size and/or star formation rate. There are important consequences for global feedback effects in galaxies: field stars should therefore contribute proportionately to the volume of the warm ionized medium, and equal relative contributions by superbubbles of all sizes to the interstellar porosity are expected.
\end{abstract}

Subject headings: stars: early-type — stars: formation — stars: statistics — galaxies: star clusters — galaxies: stellar content - galaxies: individual (SMC)

\section{INTRODUCTION}

It is commonly held that most massive, OB stars are found in stellar clusters, or associations, since their short $(\sim 10 \mathrm{Myr})$ lifetimes are not long enough to permit spatial dispersion from their natal companions. However, apparently-isolated, massive field stars are well-known to exist, including a class of runaway OB stars with unusually large $\left(\gtrsim 30 \mathrm{~km} \mathrm{~s}^{-1}\right)$ velocities. While runaway OB stars are generally believed to be kinematically ejected from a parent $\mathrm{OB}$ association, the ordinary non-runaway field OB stars have been suggested to originate in a different mode of star formation from their counterparts in associations. Suggested differences between clusters and the field in the stellar initial mass function (IMF) for high-mass stars (Massey 2002; Kroupa \& Weidner 2003) support this possibility. This contribution explores the relationship between OB associations and massive field stars.

In recent years, it has emerged that the number of stars $N_{*}$ per cluster appears to follow a universal power law distribution:

$$
N\left(N_{*}\right) d N_{*} \propto N_{*}^{-2} d N_{*} \quad .
$$

This has been found empirically for young, massive clusters (e.g., Hunter et al. 2003; Zhang \& Fall 1999), super star clusters (Meurer et al. 1995), globular clusters (e.g., Harris \& Pudritz 1994) and H II regions (Oey \& Clarke 1998). The apparent universality of this relation is emerging as fundamental (e.g., Oey \& Muñoz-Tuñon 2003; Oey \& Clarke 1998; Elmegreen \& Efremov 1997), similar to the constant power-law relation for the IMF. If individual, field OB stars have a fundamentally different origin from clustered OB stars, then this is likely to be manifest in the distribution of $N_{*}$, near $N_{*}=1$, where $N_{*}$ specifically counts massive stars only (here, $m \gtrsim 10 \mathrm{M}_{\odot}$ ). In what follows, we examine the form of $N\left(N_{*}\right)$, for small $N_{*}$, a regime that has not been investigated to date, to illuminate the relationship between massive star clustering and massive field stars.

\section{THE SMC SAMPLE OF OB STARS}

A study of OB star clustering properties and field stars requires high spatial resolution and essentially complete detection of the $\mathrm{OB}$ stars over a large area of a given galaxy. The Magellanic Clouds are optimal target galaxies by virtue of their proximity and high Galactic latitude. Indeed, some of the earliest work on $\mathrm{OB}$ associations and massive star censuses was carried out for the Magellanic Clouds by Feast et al. (1960), Sanduleak (1969), Lucke \& Hodge (1970), Azzopardi \& Vigneau (1982), and Hodge (1985). More recently, the $U B V R$ survey data of Massey (2002) for the Small Magellanic Cloud (SMC), together with UV photometry (Parker et al. 1998) from the Ultraviolet Imaging Telescope (UIT), provide unprecedented depth, resolution, completeness, and broadband coverage for the massive star population over a large fraction of the SMC. The area observed by UIT is smaller than the optical survey, but covers the SMC bar, which includes most of the active star formation in that galaxy. Figure 1 shows the regions covered by the two surveys. SMC OB associations 


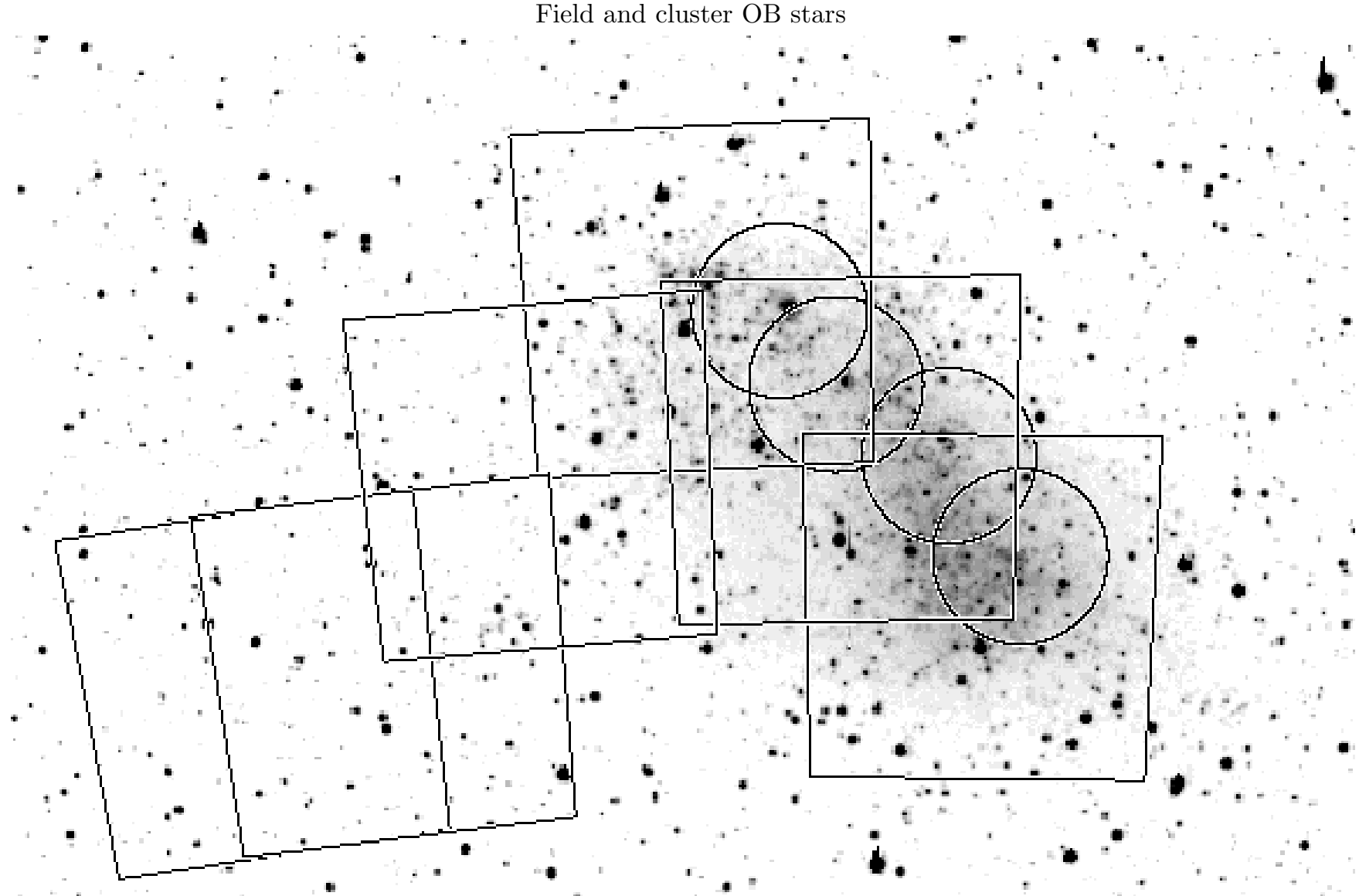

FIG. 1.- Grayscale $R$-band image of the SMC (Bothun \& Thompson 1988) showing the area coverage for the $U B V R$ survey (Massey et al. 2002; rectangular regions) and the UIT survey (Parker et al. 1998; circular regions). The optical fields are $79^{\prime}$ square, and the UIT fields are $37^{\prime}$ in diameter. North is up, east to the left.

(1985) and Battinelli (1991), the latter from the photographic OB census of Azzopardi \& Vigneau (1982). Here, we re-examine the clustering properties of these massive stars in the SMC, using the modern datasets to first systematically identify OB stars, and then to systematically identify groups or associations of them. We will especially focus on the statistical properties of the low- $N_{*}$ regime.

Parker et al. (1998) provide UIT photometry in the B5 filter $\left(\lambda_{\text {eff }}=1615 \AA, \Delta \lambda=225 \AA\right)$, which recently has been recalibrated by Parker, Cornett, \& Stecher (2004, in preparation). The $U B V R$ photometry of Massey (2002) was observed through a Harris filter set and transformed to the Johnson-Cousins system (Landolt 1992). Reddeningfree $Q$ indices were computed using the extinction law of Cardelli, Clayton, \& Mathis (1989) with a total to selective extinction ratio $R_{V}=3.1$. Different combinations of filters were used to best select two different stellar samples: Stars with initial masses $\gtrsim 10 \mathrm{M}_{\odot}$, corresponding to spectral types of B0 V, B0.5 I and earlier, were selected on the basis of their optical photometry only; and stars with initial masses $\gtrsim 20 \mathrm{M}_{\odot}$, corresponding to spectral types of O9 V, B0 I and earlier were selected on the basis of both their $U B V R$ and UIT photometry. We refer to the former as the OB sample, and the latter as the O-star sample, although we emphasize that neither sample consists of spectroscopically confirmed stars. Our criteria are as follows, using an SMC distance modulus $\mathrm{DM}=18.88$ (Dolphin et al. 2001) and global average extinction $A_{B}=0.53$ :

$$
m_{B} \leq-4.2+\mathrm{DM}+A_{B}
$$

$$
\leq 15.21
$$$$
Q_{U-R, B-R} \leq-0.84,
$$$$
Q_{B 5-U, B-R} \leq-1.45,
$$$$
Q_{B 5-V, U-V} \leq 0.03 \text {, }
$$

where

$$
\begin{aligned}
Q_{U-R, B-R} & =\left(m_{U}-m_{R}\right)-\frac{A_{U}-A_{R}}{A_{B}-A_{R}}\left(m_{B}-m_{R}\right) \\
& =\left(m_{U}-m_{R}\right)-1.396\left(m_{B}-m_{R}\right), \\
Q_{B 5-U, B-R} & =\left(m_{B 5}-m_{U}\right)-\frac{A_{B 5}-A_{U}}{A_{B}-A_{R}}\left(m_{B}-m_{R}\right) \\
& =\left(m_{B 5}-m_{U}\right)-1.668\left(m_{B}-m_{R}\right), \\
Q_{B 5-V, U-V} & =\left(m_{B 5}-m_{V}\right)-\frac{A_{B 5}-A_{V}}{A_{U}-A_{V}}\left(m_{U}-m_{V}\right) \\
& =\left(m_{B 5}-m_{V}\right)-2.718\left(m_{U}-m_{V}\right) .
\end{aligned}
$$

The OB sample is selected from equations 2 and 3 only, while the O-star sub-sample meets all of equations 2 to 5 .

Table 1 shows all the stars having both UIT B5 photometry, $U B V R$ photometry, and spectral classifications listed by Massey (2002), excluding stars with objective prism classifications and those identified as uncertain spectral types. The ID number and classification from Massey (2002) are shown in Columns 1 and 2. Column 3 shows whether the star met the O-star selection criteria above. Our attempts to select only stars earlier than $\mathrm{O} 9 \mathrm{~V}$ and B0 I were successful for 27 out of 31 stars; of the 4 not 
selected, one had nebular emission affecting the photometry and two others had weak metal lines (designation W in spectral type). The false positives were 8 out 26 stars; the majority of the false positives were weak lined stars (W), emission line stars, and peculiar stars.

\section{IDENTIFICATION OF OB ASSOCIATIONS}

Having identified two samples of $1360 \mathrm{OB}$ and $382 \mathrm{O}$ stars, we then used the friends-of-friends algorithm described by Battinelli (1991) to identify the associations of these stars. Our parameter $N_{*}$ refers specifically to counts of OB stars from these samples, identified as described above. Battinelli's algorithm adopts as the clustering distance $d_{s}$ between associated members the value of $d_{s}$ that maximizes the number of clusters for $N_{*} \geq 3$. All stars within $d_{s}$ of another member star are defined to be within the same group. (We do not distinguish between "group," "association," and "cluster" in this work.) Figure 2 shows the number of clusters $N$ as a function $d_{s}$ near the peak in $N$ for the OB sample (Figure $2 a$ ) and O-star sample (Figure $2 b)$. For the OB sample, the characteristic $d_{s}=28$ pc $\left(97^{\prime \prime}\right)$ is straightforward to determine, but for the Ostar sample, it is apparent that $N$ is less sensitive to $d_{s}$. We adopt $d_{s}=34 \mathrm{pc}\left(117^{\prime \prime}\right)$ for the O-star sample, corresponding to the mean value over the considered range; the larger value of $d_{s}$ as compared to the $\mathrm{OB}$ sample is consistent with the lower stellar density for O stars. Although the clustering properties are relatively insensitive to the choice of $d_{s}$ within a few pc, we caution that larger values of $d_{s}$ will result in flatter slopes for the clustering law $N\left(N_{*}\right)$ (e.g., equation 1), as discussed below in $\S 4$. Our values of $d_{s}$ are much smaller than Battinelli's value of $60 \mathrm{pc}$; this is consistent with a much greater completeness in our data, yielding a higher density of OB stars, and therefore smaller clustering distance.

Table 2 presents the groups having at least 3 stars that are identified from our OB sample. Column 1 shows the group ID number, columns 2 and 3 give the group centroid position in decimal degrees (J2000.0), columns 4 and 5 give the group diameter $D$ in arcmin and pc, respectively, and column 6 lists the number of stars in the group. Table 3 presents the groups identified from the O star sample in the same way. The group diameters are defined as $D=\frac{1}{2}(\Delta \alpha+\Delta \delta)$ following Battinelli (1991), where $\Delta \alpha$ and $\Delta \delta$ represent the maximum difference between members in RA and Dec, respectively, in degrees of arc. We also list the individual member stars for each group in Tables 4 and 5, which are fully available in the on-line edition. The first two columns of Tables 4 and 5 give the RA and Dec of each star in decimal degrees (J2000.0), columns 3 - 10 give the $U B V R$ magnitudes and uncertainties from Massey (2002), and the last two columns give the star ID from Massey (2002) and our newly-determined OB group ID. Table 5 also lists the UIT B5 magnitudes from Parker et al. (2004) in columns 11 and 12. Note that these group identifications represent the results from two separate runs of the group-finding algorithm; thus, if a star belongs to both samples, it may belong to groups in both Table 4 and Table 5.

Figure 3 shows the location of the group centroids compared to those of associations identified by Hodge (1985; solid black circles) and Battinelli (1991; dashed black circles). Our OB and O-star groups are indicated by the solid blue and red circles, respectively. The circle sizes correspond to the mean diameters $D$ from Tables 2 and 3 . For the Hodge objects, the diameters are taken to be the mean of the dimensions in RA and Dec given by Hodge (1985). There is good general correspondence between the positions of our groups and these earlier catalogs; certainly at least as good as the correspondence between the Hodge (1985) and Battinelli (1991) identifications. It is apparent that the net tendency from our smaller $d_{s}$ is for our associations to be smaller than those in the earlier catalogs, and to break up some of those associations into smaller groups. Figures $4 a, c$ and $4 b, d$ show the size distributions for, respectively, the OB and O-star samples. It is apparent that, aside from individual stars, the peak in the distribution is around 10-15 pc, compared to $50 \mathrm{pc}$ for both the Battinelli (1991) and Hodge (1985) catalogs. Whereas Hodge (1985) reports that the mean diameter of his sample is similar to the mean for the LMC sample, our smaller characteristic value is consistent with the lower luminosities of the SMC $\mathrm{H}$ II regions and star-forming regions compared to those of the LMC (e.g., Kennicutt, Edgar, \& Hodge 1989).

\section{FIELD VS CLUSTERS}

Figure 5 shows the distribution in $N_{*}$ for the OB groups (panel $a$ ) and O-star groups (panel $b$ ). Two slopes are shown fitted to the power-law distribution, weighted by the square root of the bin value, $\sqrt{N\left(\log N_{*}\right)}$ : the solid line shows the fit for the entire distribution, yielding $-2.51 \pm 0.29$ and $-2.33 \pm 0.42$ for the $\mathrm{OB}$ and O-star samples, respectively; and the dotted line shows the fit omitting the first bin, which corresponds to single stars, resulting in fits of $-2.27 \pm 0.38$ and $-2.11 \pm 0.55$ for the $\mathrm{OB}$ and O-star samples, respectively. The fits omitting the single stars agree with the power-law slope of -2 (equation 1 ), found for the $N_{*}$ distribution in a variety of systems, as discussed in $\S 1$. We again emphasize, as mentioned above, that the fitted slope has a dependence on the clustering distance $d_{s}$ : for large $d_{s}$, more stars are drawn into the associations, causing the resultant slope of the clustering law to be flatter relative to clustering defined by a small $d_{s}$. Since we seek a characteristic value for the slope of $N\left(N_{*}\right)$, it is important to ensure that $d_{s}$ is in turn characteristic of the sample. For the $d_{s}$ in the extreme range of $20-$ $40 \mathrm{pc}$ (see Figure $2 b$ ), the O-star sample shows a variation in fitted slope, omitting the field stars, of $-2.79 \pm 0.84$ to $-1.85 \pm 0.52$, respectively.

It is apparent that the single stars are slightly greater in number than the power law for the remainder of the $N_{*}$ distribution, particularly in the OB sample. How significant is this excess in the isolated, "field" stars? We note that the magnitude of the excess may be a lower limit, since the slope of the $N_{*}$ distribution is expected to flatten slightly at the smallest values of $N_{*}$, owing to statistical effects, as follows. We assume, as suggested above, that the OB stars counted in the value of $N_{*}$ represent only the most massive stars for a population of star clusters that are in reality populated by a mass distribution described by a conventional IMF. Since we identified only OB stars, our clusters are selected with the criterion that each cluster contains a star of at least mass $m_{\text {cut }}$. The smallest, "unsaturated" clusters have a lower probability of having their maximum stellar mass $m_{\max } \geq m_{\text {cut }}$, so they will be 
TABLE 1

COMPARISON OF O-STAR CRITERIA

\begin{tabular}{|c|c|c|c|c|c|}
\hline Cat ID $^{\mathrm{a}}$ & Sp Type ${ }^{b}$ & $\begin{array}{l}\text { Identified }^{\mathrm{c}} \\
(\mathrm{y} \text { or } \mathrm{n})\end{array}$ & Cat ID ${ }^{a}$ & Sp Type ${ }^{b}$ & $\begin{array}{l}\text { Identified }^{\mathrm{C}} \\
(\mathrm{y} \text { or } \mathrm{n})\end{array}$ \\
\hline 16828 & O5 III(f) & $\mathrm{y}$ & 53382 & B0 IIWW & $\mathrm{y}$ \\
\hline 38024 & $\mathrm{O} 5 \mathrm{~V}$ & $\mathrm{y}$ & 54456 & B0 IW & $\mathrm{y}$ \\
\hline 5869 & O7 V & $\mathrm{y}$ & 54958 & B0 IIIWW & $\mathrm{y}$ \\
\hline 16056 & O7 III & $\mathrm{y}$ & 9079 & B1.5 V & $\mathrm{n}$ \\
\hline 16885 & O7 III & $\mathrm{y}$ & 9488 & B1 I & $\mathrm{n}$ \\
\hline 17457 & O7 III & $\mathrm{y}$ & 18700 & B1 Ib & $\mathrm{y}$ \\
\hline 17927 & O7.5Iaf+ & $\mathrm{y}$ & 37732 & B1 IIW & $\mathrm{n}$ \\
\hline 19650 & O7.5 III & $\mathrm{y}$ & 42740 & B1 III & $\mathrm{n}$ \\
\hline 22837 & O7.5 V & $\mathrm{y}$ & 43807 & B1 V & $\mathrm{n}$ \\
\hline 40341 & O7 III & $\mathrm{y}$ & 44784 & B1 III & $\mathrm{n}$ \\
\hline 43724 & $\mathrm{O} 7 \mathrm{~V}$ & $\mathrm{y}$ & 45114 & B1 III & $\mathrm{y}$ \\
\hline 45521 & O7 If & $\mathrm{y}$ & 45438 & B1 Ve & $\mathrm{n}$ \\
\hline 46035 & O7 III & $\mathrm{y}$ & 45809 & B1 III & $\mathrm{n}$ \\
\hline 52170 & O7 V & $\mathrm{y}$ & 50609 & B1 III & $\mathrm{n}$ \\
\hline 27731 & $08.5 \mathrm{~V}$ & $\mathrm{y}$ & 51009 & B1.5 III & $\mathrm{n}$ \\
\hline 43197 & $08.5 \mathrm{~V}$ & $\mathrm{y}$ & 53225 & B1 V & $\mathrm{n}$ \\
\hline 43734 & $\mathrm{O} 8 \mathrm{~V}$ & $\mathrm{n}$ & 26901 & B2 IIIW & $\mathrm{y}$ \\
\hline 49580 & $08.5 \mathrm{~V}$ & $\mathrm{y}$ & 32907 & B2 II & $\mathrm{n}$ \\
\hline 50825 & O8 Vn & $\mathrm{y}$ & 50826 & B2.5 III & $\mathrm{n}$ \\
\hline 53373 & $08.5 \mathrm{~V}$ & $\mathrm{y}$ & 54818 & B2 I & $\mathrm{n}$ \\
\hline 6406 & O9+NEB & $\mathrm{n}$ & 24929 & B3 I & $\mathrm{n}$ \\
\hline 11777 & O9 II & $\mathrm{y}$ & 43844 & B3 I & $\mathrm{n}$ \\
\hline 40610 & O9 V & $\mathrm{y}$ & 44828 & B3 I & $\mathrm{n}$ \\
\hline 45677 & O9 III & $\mathrm{y}$ & 47028 & B3 I & $\mathrm{n}$ \\
\hline 47540 & O9 III & $\mathrm{y}$ & 49606 & B3 I & $\mathrm{n}$ \\
\hline 3459 & B0 IWW & $\mathrm{n}$ & 45722 & $\mathrm{~B} 8 \mathrm{Ve}$ & $\mathrm{y}$ \\
\hline 13075 & B0.5 III & $\mathrm{y}$ & 50475 & B8 Iab & $\mathrm{n}$ \\
\hline 13831 & B0.5 V & $\mathrm{y}$ & 53084 & B8-A0 I & $\mathrm{n}$ \\
\hline 15503 & B0.5 V & $\mathrm{n}$ & 5391 & B pec & $\mathrm{y}$ \\
\hline 15742 & B0 IIWW & $\mathrm{y}$ & 21801 & В pec & $\mathrm{y}$ \\
\hline 18614 & B0 IIW & $\mathrm{n}$ & 24914 & B extr & $\mathrm{y}$ \\
\hline 20656 & B0 I & $\mathrm{y}$ & 40851 & B extr & $\mathrm{y}$ \\
\hline 23702 & B0 IW & $\mathrm{y}$ & 10915 & A0 Ia & $\mathrm{n}$ \\
\hline 41648 & B0.5 III & $\mathrm{n}$ & 43215 & A0 I & $\mathrm{n}$ \\
\hline 43686 & B0.5 V & $\mathrm{n}$ & 48732 & A0 I & $\mathrm{n}$ \\
\hline 43758 & B $0.2 \mathrm{~V}$ & $\mathrm{n}$ & 52992 & A1 I & $\mathrm{n}$ \\
\hline 51575 & B0 III & $\mathrm{y}$ & $\ldots$ & $\ldots$ & $\ldots$ \\
\hline
\end{tabular}

${ }^{a}$ Catalog number from Massey (2002)

${ }^{\mathrm{b}}$ Spectroscopic type compiled by Massey (2002)

${ }^{\mathrm{c}}$ Identified by O-star criteria equations $2-5$ 

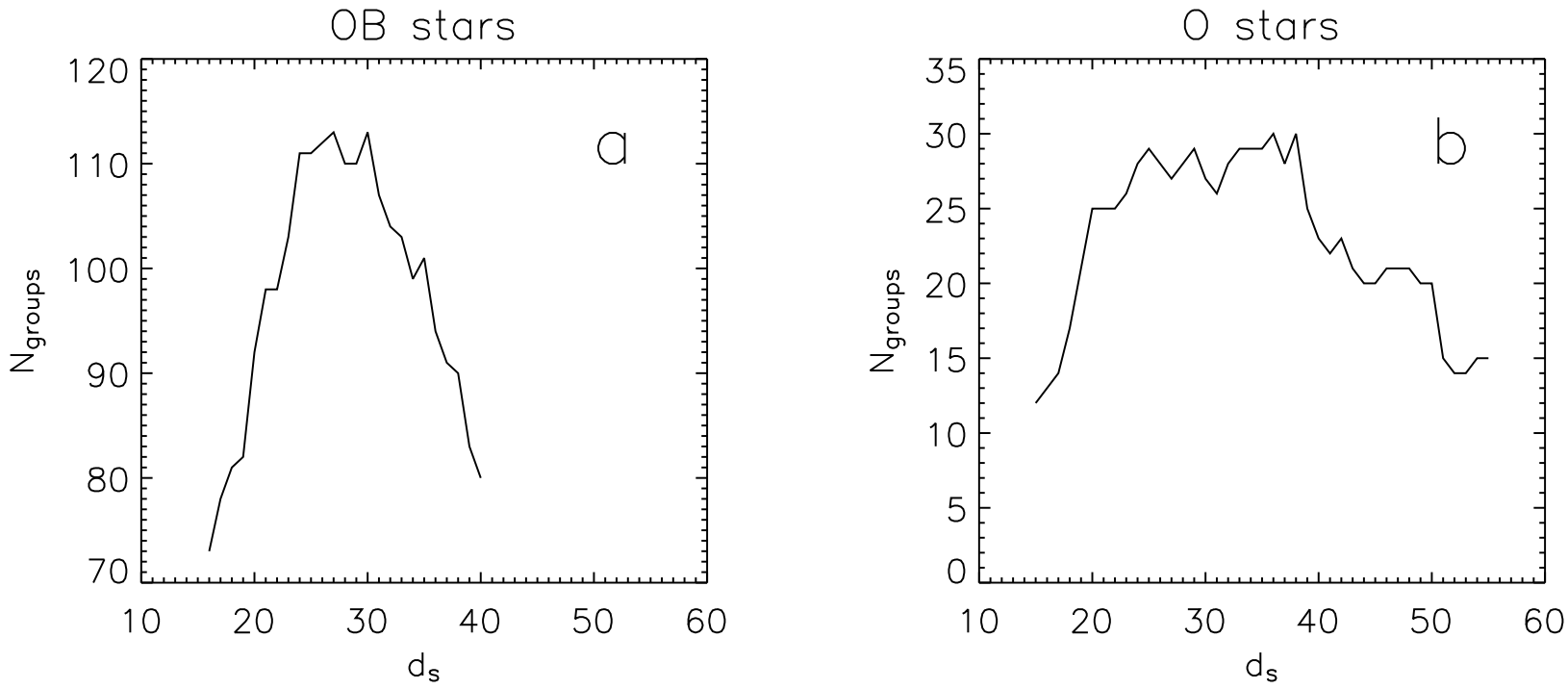

Fig. 2.- Number of groups with $N_{*} \geq 3$ as a function of clustering distance $d_{s}$ for the OB sample (panel $a$ ) and O-star sample (panel $b$ ).

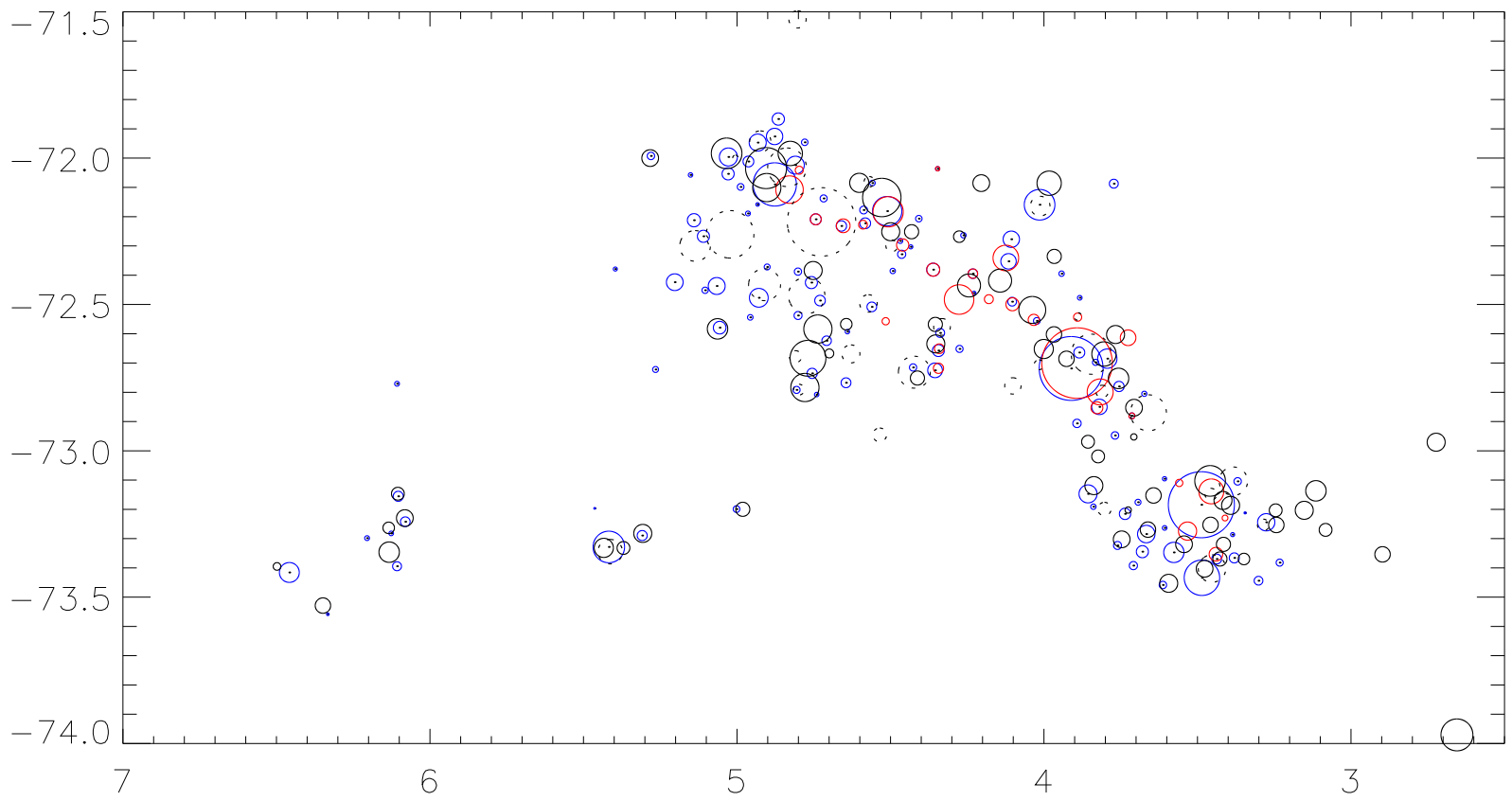

FIG. 3.- Centroids and sizes of associations identified by Hodge (1985; solid black), Battinelli (1991; dotted black), our OB sample (blue) and our O-star sample (red). Note that all blue circles have a dot at the center, thus overplotted identical circles can be identified by small dots in the center of red circles. (RA and Dec given in degrees of arc.) 
Field and cluster OB stars

TABLE 2

Groups from the CANDidate OB SAmple

\begin{tabular}{|c|c|c|c|c|c|}
\hline OB grp & RA & Dec & $D(\min )$ & $D(\mathrm{pc})$ & $N_{*}$ \\
\hline 20 & 11.3029 & -73.3820 & 1.38 & 23.93 & 4 \\
\hline 24 & 11.3639 & -73.2441 & 3.39 & 58.90 & 5 \\
\hline 29 & 11.5932 & -73.1044 & 1.45 & 25.21 & 4 \\
\hline 30 & 11.5857 & -73.4439 & 1.69 & 29.37 & 3 \\
\hline 31 & 11.5817 & -73.2120 & 0.38 & 6.64 & 3 \\
\hline 38 & 12.0545 & -73.1842 & 12.91 & 224.68 & 70 \\
\hline 40 & 11.7724 & -73.2863 & 0.81 & 14.05 & 3 \\
\hline 41 & 11.8078 & -73.3658 & 1.97 & 34.36 & 4 \\
\hline 45 & 12.0111 & -73.3695 & 1.92 & 33.33 & 4 \\
\hline 47 & 12.2249 & -73.4337 & 6.93 & 120.56 & 26 \\
\hline 64 & 12.2657 & -72.0873 & 1.75 & 30.42 & 4 \\
\hline 73 & 12.4821 & -73.3470 & 3.95 & 68.70 & 16 \\
\hline 74 & 12.4058 & -73.0955 & 0.85 & 14.84 & 3 \\
\hline 75 & 12.4251 & -72.8051 & 1.02 & 17.81 & 3 \\
\hline 86 & 12.5262 & -73.2635 & 0.90 & 15.73 & 3 \\
\hline 91 & 12.6156 & -72.8806 & 1.16 & 20.18 & 4 \\
\hline 98 & 12.6898 & -73.4584 & 1.47 & 25.53 & 6 \\
\hline 99 & 12.7466 & -72.6852 & 3.78 & 65.71 & 9 \\
\hline 100 & 12.6862 & -72.7800 & 1.99 & 34.61 & 5 \\
\hline 101 & 12.7490 & -73.2845 & 3.42 & 59.56 & 10 \\
\hline 104 & 12.7620 & -73.1756 & 1.14 & 19.90 & 4 \\
\hline 107 & 13.1672 & -72.7186 & 12.48 & 217.09 & 55 \\
\hline 110 & 12.8384 & -73.3445 & 2.35 & 40.93 & 7 \\
\hline 112 & 12.8514 & -72.9475 & 1.46 & 25.40 & 3 \\
\hline 115 & 12.8844 & -72.6976 & 1.20 & 20.94 & 3 \\
\hline 118 & 12.9530 & -72.8498 & 3.02 & 52.62 & 7 \\
\hline 119 & 12.8984 & -72.4770 & 0.91 & 15.80 & 3 \\
\hline 120 & 12.9375 & -73.2152 & 2.21 & 38.45 & 8 \\
\hline 121 & 12.9762 & -73.3923 & 1.59 & 27.72 & 3 \\
\hline 123 & 13.1031 & -72.1594 & 5.99 & 104.24 & 15 \\
\hline 125 & 13.0386 & -72.6643 & 2.14 & 37.23 & 6 \\
\hline 131 & 13.0357 & -72.3948 & 1.05 & 18.26 & 3 \\
\hline 137 & 13.1042 & -73.3233 & 1.57 & 27.33 & 4 \\
\hline 142 & 13.3029 & -73.1472 & 3.52 & 61.32 & 8 \\
\hline 150 & 13.2429 & -72.9064 & 1.64 & 28.61 & 3 \\
\hline 152 & 13.2756 & -73.1909 & 1.06 & 18.42 & 3 \\
\hline 158 & 13.4218 & -72.5561 & 1.30 & 22.63 & 3 \\
\hline 160 & 13.4898 & -72.2771 & 3.20 & 55.66 & 6 \\
\hline 170 & 13.5753 & -72.3525 & 3.01 & 52.33 & 9 \\
\hline 174 & 13.6399 & -72.4905 & 1.70 & 29.66 & 4 \\
\hline 195 & 13.9908 & -72.3949 & 1.86 & 32.43 & 4 \\
\hline 197 & 13.9913 & -72.2634 & 1.06 & 18.43 & 3 \\
\hline 204 & 14.0291 & -72.4610 & 0.71 & 12.40 & 3 \\
\hline 211 & 14.0923 & -72.0363 & 0.82 & 14.26 & 3 \\
\hline 228 & 14.3369 & -72.6518 & 1.37 & 23.86 & 5 \\
\hline 233 & 14.4076 & -72.3812 & 2.52 & 43.93 & 5 \\
\hline 235 & 14.4236 & -72.2069 & 1.26 & 22.01 & 3 \\
\hline 237 & 14.5052 & -72.5974 & 1.74 & 30.22 & 5 \\
\hline 241 & 14.5732 & -72.6575 & 2.34 & 40.66 & 8 \\
\hline 244 & 14.7403 & -72.1810 & 5.74 & 99.90 & 42 \\
\hline 247 & 14.6652 & -72.7249 & 2.85 & 49.57 & 5 \\
\hline 248 & 14.5854 & -72.3032 & 0.78 & 13.66 & 3 \\
\hline 255 & 14.6828 & -72.2829 & 0.98 & 17.03 & 3 \\
\hline 256 & 14.7037 & -72.3284 & 1.59 & 27.67 & 3 \\
\hline 263 & 14.8208 & -72.0852 & 1.10 & 19.18 & 3 \\
\hline 265 & 14.8466 & -72.3857 & 1.06 & 18.47 & 3 \\
\hline 270 & 14.8983 & -72.7153 & 1.40 & 24.44 & 3 \\
\hline 277 & 15.0082 & -72.2228 & 2.09 & 36.34 & 4 \\
\hline 279 & 14.9884 & -72.1773 & 1.54 & 26.76 & 3 \\
\hline 294 & 15.1744 & -72.5081 & 1.87 & 32.59 & 4 \\
\hline
\end{tabular}


TABle 2

(continued)

\begin{tabular}{|c|c|c|c|c|c|}
\hline OB grp & RA & Dec & $D(\min )$ & $D(\mathrm{pc})$ & $N_{*}$ \\
\hline 303 & 15.2701 & -72.2317 & 2.02 & 35.18 & 4 \\
\hline 315 & 15.3803 & -72.1378 & 1.38 & 23.95 & 3 \\
\hline 325 & 15.4218 & -71.9461 & 1.28 & 22.21 & 3 \\
\hline 327 & 15.5244 & -72.2091 & 2.15 & 37.40 & 5 \\
\hline 331 & 15.5112 & -72.5928 & 0.88 & 15.30 & 3 \\
\hline 333 & 15.5852 & -72.0239 & 3.47 & 60.41 & 7 \\
\hline 337 & 15.8589 & -72.0899 & 8.45 & 147.10 & 79 \\
\hline 340 & 15.6331 & -71.8665 & 2.41 & 41.86 & 5 \\
\hline 344 & 15.7216 & -71.9261 & 3.20 & 55.77 & 4 \\
\hline 345 & 15.6789 & -72.7675 & 1.92 & 33.33 & 4 \\
\hline 347 & 15.7562 & -72.4252 & 2.35 & 40.82 & 6 \\
\hline 348 & 15.7160 & -72.4867 & 2.07 & 35.98 & 4 \\
\hline 351 & 15.7653 & -72.6240 & 1.86 & 32.41 & 3 \\
\hline 354 & 15.9167 & -71.9469 & 3.32 & 57.77 & 8 \\
\hline 357 & 15.8683 & -72.3877 & 1.43 & 24.85 & 3 \\
\hline 365 & 16.0226 & -72.7355 & 2.00 & 34.80 & 8 \\
\hline 366 & 15.9992 & -72.5378 & 1.54 & 26.85 & 4 \\
\hline 367 & 16.0374 & -72.8077 & 0.89 & 15.50 & 3 \\
\hline 369 & 16.0700 & -72.0117 & 2.11 & 36.70 & 4 \\
\hline 373 & 16.1001 & -72.1584 & 0.70 & 12.26 & 3 \\
\hline 376 & 16.1858 & -72.3718 & 1.15 & 20.02 & 3 \\
\hline 377 & 16.2677 & -71.9959 & 3.51 & 60.99 & 19 \\
\hline 382 & 16.2279 & -72.0984 & 1.30 & 22.70 & 4 \\
\hline 383 & 16.2297 & -72.1892 & 0.95 & 16.61 & 3 \\
\hline 385 & 16.2451 & -72.7920 & 1.36 & 23.67 & 5 \\
\hline 388 & 16.3209 & -72.0536 & 2.35 & 40.90 & 4 \\
\hline 391 & 16.3701 & -72.4773 & 3.66 & 63.75 & 8 \\
\hline 399 & 16.5235 & -72.5441 & 1.08 & 18.87 & 4 \\
\hline 415 & 16.7752 & -72.2671 & 2.34 & 40.67 & 5 \\
\hline 416 & 16.7213 & -72.0574 & 0.89 & 15.42 & 3 \\
\hline 420 & 16.8253 & -72.2122 & 2.59 & 45.08 & 5 \\
\hline 421 & 16.7891 & -72.4370 & 3.31 & 57.54 & 6 \\
\hline 426 & 16.8895 & -72.5793 & 2.48 & 43.11 & 5 \\
\hline 429 & 16.9275 & -72.4516 & 1.23 & 21.39 & 4 \\
\hline 439 & 17.0803 & -71.9925 & 1.43 & 24.93 & 3 \\
\hline 444 & 17.2285 & -72.4238 & 3.28 & 56.99 & 10 \\
\hline 449 & 17.3037 & -73.1992 & 1.29 & 22.40 & 4 \\
\hline 460 & 17.7278 & -72.7223 & 1.18 & 20.45 & 3 \\
\hline 464 & 17.8254 & -72.3788 & 0.79 & 13.72 & 4 \\
\hline 480 & 18.4627 & -73.2896 & 1.96 & 34.18 & 8 \\
\hline 486 & 18.8834 & -73.3285 & 6.18 & 107.50 & 24 \\
\hline 496 & 18.9001 & -73.1967 & 0.32 & 5.62 & 3 \\
\hline 529 & 20.6192 & -72.7708 & 0.96 & 16.66 & 3 \\
\hline 538 & 21.0626 & -73.1555 & 1.95 & 33.88 & 8 \\
\hline 539 & 21.0881 & -73.2422 & 1.88 & 32.75 & 4 \\
\hline 546 & 21.2965 & -73.2821 & 0.90 & 15.68 & 3 \\
\hline 547 & 21.3705 & -73.3945 & 1.73 & 30.09 & 4 \\
\hline 550 & 21.5905 & -73.2984 & 0.96 & 16.65 & 3 \\
\hline 568 & 22.3738 & -73.5585 & 0.52 & 8.98 & 3 \\
\hline 569 & 22.6252 & -73.4156 & 3.88 & 67.59 & 11 \\
\hline 576 & 22.6252 & -73.4156 & 3.88 & 67.59 & 11 \\
\hline
\end{tabular}


TABLE 3

Groups From the CANDidate O-STAR SAMPle

\begin{tabular}{rcccrr}
\hline \hline O grp & RA & Dec & $D(\min )$ & $D(\mathrm{pc})$ & $N_{*}$ \\
\hline 8 & 11.9117 & -73.1385 & 4.87 & 84.71 & 11 \\
11 & 11.8196 & -73.2295 & 1.11 & 19.35 & 3 \\
13 & 12.0083 & -73.3533 & 2.64 & 45.86 & 4 \\
19 & 12.2742 & -73.2744 & 3.57 & 62.11 & 6 \\
24 & 12.2523 & -73.1099 & 1.41 & 24.49 & 3 \\
26 & 12.4694 & -72.6139 & 3.03 & 52.78 & 4 \\
38 & 12.6156 & -72.8806 & 1.16 & 20.18 & 4 \\
41 & 13.0895 & -72.7004 & 13.76 & 239.45 & 57 \\
45 & 12.9077 & -72.7988 & 5.06 & 88.03 & 12 \\
50 & 12.9830 & -72.8530 & 2.30 & 40.07 & 4 \\
51 & 12.9693 & -72.5435 & 1.60 & 27.84 & 4 \\
62 & 13.4522 & -72.5525 & 2.19 & 38.11 & 4 \\
67 & 13.5963 & -72.3412 & 5.02 & 87.31 & 12 \\
68 & 13.6438 & -72.4994 & 2.52 & 43.85 & 5 \\
76 & 13.8855 & -72.4820 & 1.74 & 30.28 & 3 \\
78 & 13.9908 & -72.3949 & 1.86 & 32.43 & 4 \\
81 & 14.2089 & -72.4833 & 5.75 & 100.05 & 11 \\
84 & 14.0923 & -72.0363 & 0.82 & 14.26 & 3 \\
97 & 14.4076 & -72.3812 & 2.52 & 43.93 & 5 \\
102 & 14.5581 & -72.6512 & 1.87 & 32.61 & 4 \\
104 & 14.7329 & -72.1834 & 5.90 & 102.61 & 26 \\
107 & 14.6211 & -72.7179 & 2.00 & 34.71 & 3 \\
109 & 14.6682 & -72.2968 & 2.38 & 41.43 & 5 \\
121 & 15.0359 & -72.2274 & 1.72 & 29.92 & 3 \\
124 & 15.0641 & -72.5572 & 1.42 & 24.74 & 3 \\
130 & 15.2501 & -72.2311 & 2.68 & 46.59 & 5 \\
143 & 15.5244 & -72.2091 & 2.15 & 37.40 & 5 \\
145 & 15.5583 & -72.0413 & 1.55 & 26.96 & 3 \\
146 & 15.7173 & -72.1075 & 5.36 & 93.19 & 17 \\
\hline & & & & &
\end{tabular}

TABLE 4

Group MEMBERShIP FOR THE CANDIDATE OB SAMPLE

\begin{tabular}{cccccccccccc}
\hline \hline RA & Dec & $U$ & $U$ err & $B$ & $B$ err & $V$ & $V$ err & $R$ & $R$ err & ID & OB grp \\
\hline 10.1171 & -73.5425 & 14.01 & 0.05 & 14.96 & 0.04 & 15.00 & 0.03 & 14.96 & 0.07 & 107 & 1 \\
10.1832 & -73.4063 & 14.40 & 0.05 & 15.18 & 0.04 & 15.12 & 0.03 & 14.86 & 0.07 & 298 & 2 \\
10.3880 & -73.4256 & 14.24 & 0.06 & 15.15 & 0.06 & 15.28 & 0.04 & 15.30 & 0.11 & 1037 & 3 \\
10.5417 & -73.2323 & 13.44 & 0.03 & 14.42 & 0.03 & 14.60 & 0.02 & 14.69 & 0.05 & 1600 & 4 \\
10.5515 & -73.3866 & 14.26 & 0.05 & 15.19 & 0.04 & 15.15 & 0.03 & 15.04 & 0.08 & 1631 & 5 \\
\hline
\end{tabular}

${ }^{a}$ The complete version of this table is in the electronic edition of the Journal. The printed edition contains only a sample.

TABLE 5

GRoup MEMBERSHIP FOR THE CANDIDATE O-STAR SAMPLE ${ }^{\mathrm{a}}$

\begin{tabular}{cccccccccccccc}
\hline \hline RA & Dec & $U$ & $U$ err & $B$ & $B$ err & $V$ & $V$ err & $R$ & $R$ err & B5 & B5 err & ID & O grp \\
\hline 11.1440 & -73.1597 & 13.78 & 0.05 & 14.70 & 0.04 & 14.82 & 0.03 & 14.74 & 0.07 & 11.92 & 0.05 & 4424 & 1 \\
11.2379 & -73.0130 & 13.19 & 0.03 & 14.30 & 0.03 & 14.36 & 0.02 & 14.29 & 0.04 & 11.16 & 0.09 & 4922 & 2 \\
11.2438 & -73.0213 & 14.05 & 0.05 & 14.94 & 0.04 & 14.91 & 0.03 & 14.86 & 0.07 & 12.19 & 0.05 & 4949 & 2 \\
11.3259 & -73.2564 & 12.45 & 0.02 & 13.36 & 0.01 & 13.31 & 0.01 & 13.14 & 0.02 & 10.25 & 0.08 & 5391 & 3 \\
11.3867 & -73.0773 & 13.16 & 0.04 & 14.15 & 0.04 & 14.30 & 0.03 & 14.28 & 0.06 & 10.85 & 0.06 & 5718 & 4 \\
\hline
\end{tabular}

${ }^{\text {a }}$ The complete version of this table is in the electronic edition of the Journal. The printed edition contains only a sample. 

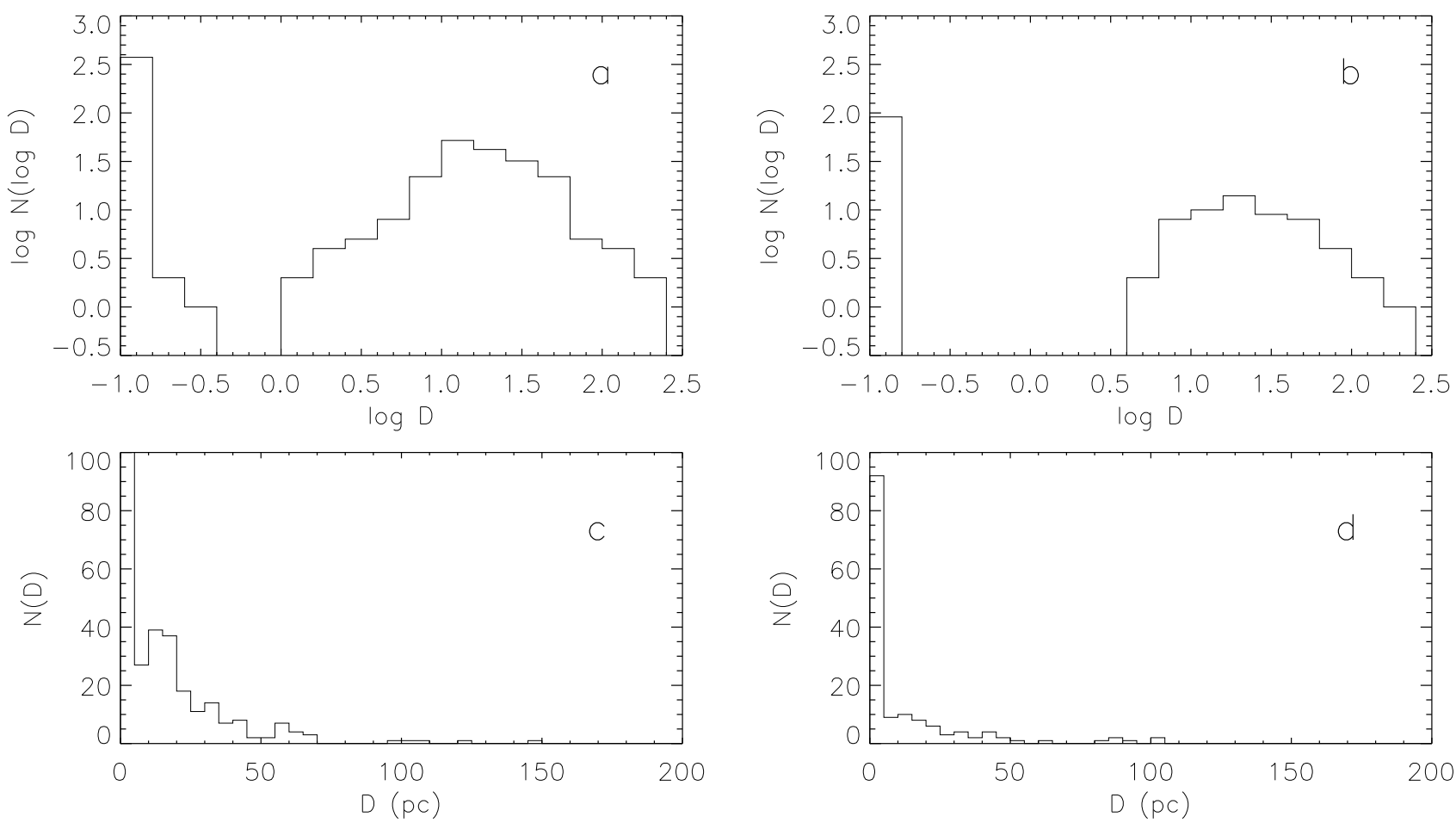

FIG. 4. - Logarithmic size distribution for groups identified from the candidate OB and O-star samples are shown in the top row (panels $a$ and $b$, respectively). The linear distributions for the OB and and O-star samples are shown in the bottom row (panels $c$ and $d$, respectively). The first bin in Figure $4 c$ has a maximum of 391.
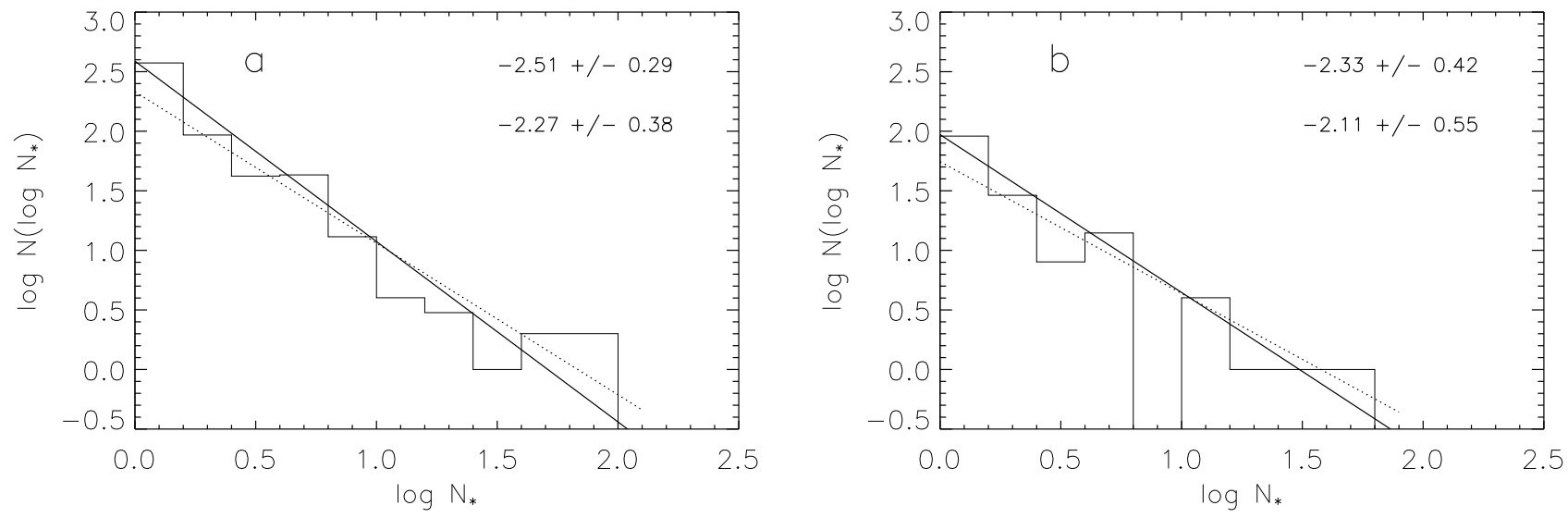

FIG. 5.- The distribution in $N_{*}$ for groups defined from the candidate OB sample (panel $a$ ) and O-star sample (panel $b$ ). Fits to the power-law exponent $(-\beta)$ are shown for the entire distributions (upper values) and omitting the first bin of single stars (lower values). Note that the slope fitted in logarithmic space is $-\beta+1$. 

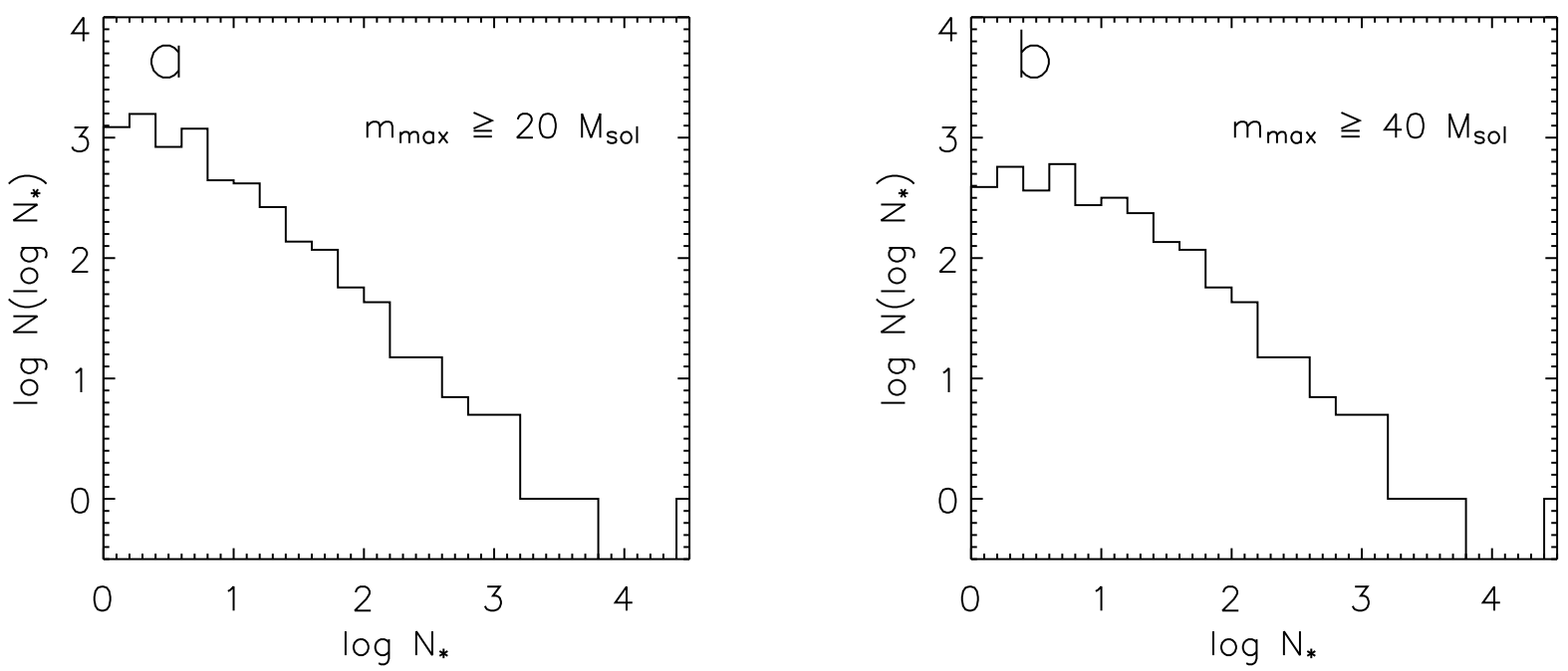

Fig. 6. - Monte Carlo models for distributions of $\log N_{*}$, selected by the criterion that the maximum stellar mass in each cluster be at least $m_{\max } \geq m_{\text {cut }}$, showing $m_{\text {cut }}=20 \mathrm{M}_{\odot}$ and $40 \mathrm{M}_{\odot}$ in panels $a$ and $b$, respectively.

progressively missing from a sample of clusters selected in this way (see Oey \& Clarke 2003 for a detailed discussion of this effect).

Figure 6 shows Monte Carlo models that demonstrate this effect. $N_{*}$ is drawn from a power-law distribution of slope -2 (equation 1 ), with the individual stellar masses drawn from a Salpeter (1955) IMF having $10 \leq m \leq$ $100 \mathrm{M}_{\odot}$. Figure $6 a$ shows the distribution in $N_{*}$ for 10,000 clusters having at least one star with mass $m_{\max } \geq 20 \mathrm{M}_{\odot}$, and Figure $6 b$ is the same, but selected for $m_{\max } \geq 40 \mathrm{M}_{\odot}$. We see a flattening in the distributions for small $N_{*}$, and the flattening is stronger in Figure $6 b$, since the probability of drawing a $40 \mathrm{M}_{\odot}$ star is less than for a $20 \mathrm{M}_{\odot}$ star.

Figure 7 shows the stellar density of all catalogued stars in the $U B V R$ survey as a function of radius around the isolated, field massive stars found in the OB (solid line) and O-star (dashed line) samples. The errors are computed as $\sqrt{n} / \pi r^{2}$ for the total $n$ stars within radius $r$, omitting the central field massive stars. As seen in Figure 7, it is apparent that the stellar density increases at small $r$. This is consistent with our suggestion that most massive field stars represent the most massive component of groups of smaller stars, as is expected from the stellar IMF.

Another effect that can offset the statistical flattening in the form of the $N_{*}$ distribution for small $N_{*}$, is evolution. In the most extreme situation, we consider that all the $\mathrm{OB}$ associations in the SMC were formed together in a single burst of global star formation. Staveley-Smith et al. (1997) find that the H I shells identified in the SMC appear to have a narrow age distribution around 5 Myr. We described above that the unsaturated, lowest- $N_{*}$ clusters have a lower average stellar mass (e.g., Oey \& Clarke 2003). Since stellar lifetimes are longer for lower-mass stars, we therefore expect that these unsaturated clusters will tend to last longer, on average, than the statistically fully-sampled, or saturated, objects. Thus, we may expect that the distribution in $N_{*}$ steepens with time.

We constructed Monte Carlo simulations of such an aging burst of clusters. Figure 8 shows the models for a population of clusters at 0,15 , and $25 \mathrm{Myr}$ after their simultaneous formation. We used the same IMF parameters are before, and the stellar ages are from the grid of Charbonnel et al. (1993) for SMC metallicity. Each of the model $N_{*}$ distributions is fitted with a power-law slope, shown in Figure 8. We see that, while it is difficult to discern the slope steepening over the entire sample after $15 \mathrm{Myr}$, and even at $25 \mathrm{Myr}$, it is apparent that the aging effect is most pronounced in the smallest $N_{*}$ bins, and that the $N_{*}=1$ bin becomes the most disproportionately enhanced. For continuous creation of the clusters, the net observed slope will be intermediate between the forms in Figure 6 and Figure 8.

The modest observed excess of single stars is also likely to be caused in part by a contribution to their population by runaway $\mathrm{OB}$ stars. If runaway $\mathrm{OB}$ stars originate in associations, however, then this contribution must be small, since runaways correspond to about $3 \%$ and $20 \%$ of field early B and early O stars, respectively (Blaauw 1961). Runaways will contribute primarily to the singlestar population, while binary runaways represent $\lesssim 19 \%$ of all runaways (Gies 1987). Binary runaways are likely to be tight pairs that remain unresolved in the $0.67 \mathrm{pc} \mathrm{px}^{-1}$ survey resolution.

It is also possible that spurious selection of candidate stars, owing to the coarse photometric criteria, are a factor. We showed in $\S 2$ that there may be a net of about $15 \%$ of the O-star sample that consist of spuriously selected B stars; while in principle, these should be distributed proportionately between clusters and the field, it is possible that crowding effects in clusters favor field selection. If so, owing to the longer lifetimes of B stars, they could contribute disproportionately to the field star population. However, we emphasize that this requires a significant variation in the spatial distribution of only the spurious candidates, between field and clusters.

The most important factor in enhancing the field star population, however, is probably the strong variation in star formation density across the SMC. The highest star 


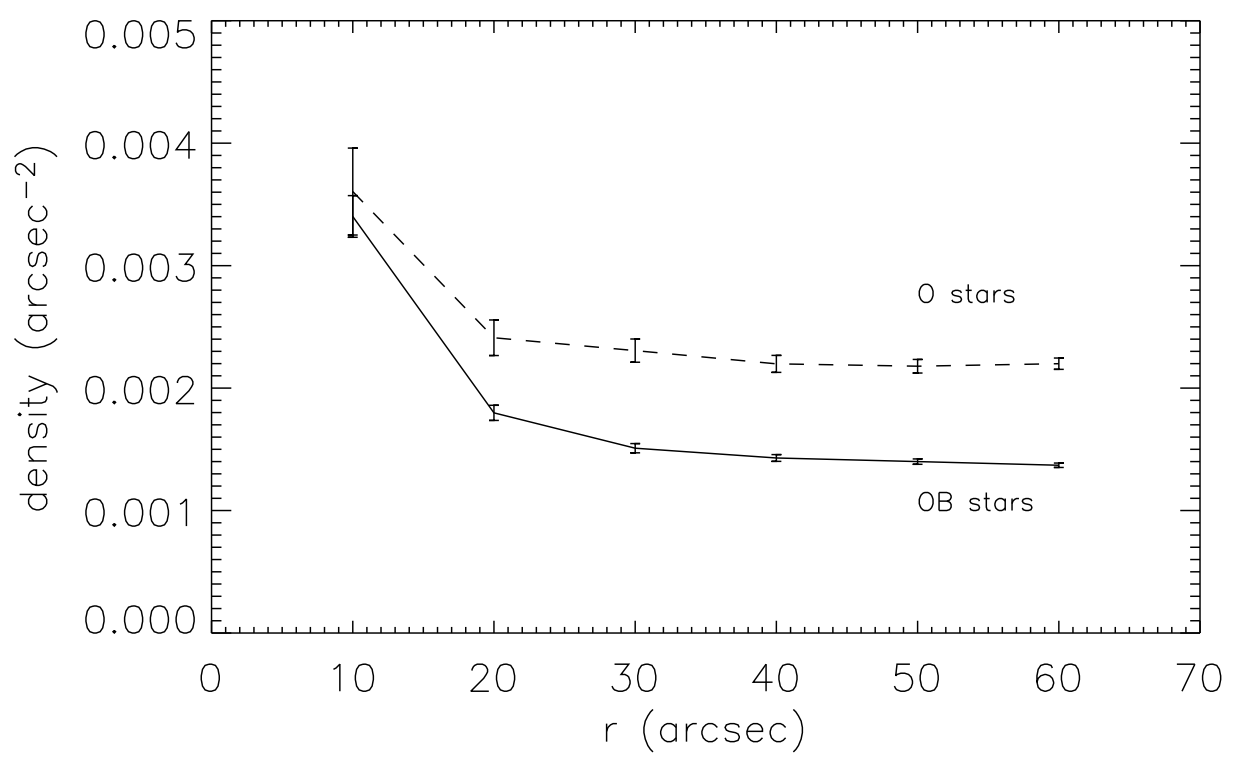

FIG. 7.- Mean stellar density $\left(\operatorname{arcsec}^{-2}\right.$ ) within radius $r$ (arcsec) from isolated massive stars in the candidate OB (solid line) and O-star (dashed line) samples $\left(10^{\prime \prime}=2.9 \mathrm{pc}\right)$. The O-star sample shows a higher background density since that sample is limited to the SMC bar region (Figure 1).

formation density occurs in the SMC bar, where, as seen in Figure 3, most of the associations are located. Individual massive field stars, on the other hand, have a much more uniform distribution, as seen in Figure 9. The uneven star formation density distribution in this galaxy therefore favors the field stars and enhances the $N_{*}=1$ bin for the OB sample in Figure 5. The effect is much less pronounced in the O-star sample, which is limited to the SMC bar region.

Considering these effects, it is apparent that there is no strong variation or change in character of the powerlaw distribution seen in Figure 5 for the smallest values of $N_{*}$. The empirical samples (Figure 5) are much smaller than those in the models and therefore have significantly poorer statistics in $\log N\left(\log N_{*}\right)$. Thus, the modeled effects will be more difficult to discern in the data. Since our results are largely consistent with a single intrinsic power-law form for the clustering law, this suggests that the massive field star population simply represents an extension of the massive cluster population extending down to $N_{*}=1$. There is no evidence that the majority of massive field stars originate from a mode of star formation that is different from those in associations. However, further studies of massive field and cluster populations in other environments are necessary to confirm the generality of these results.

\section{DISCUSSION}

For a universal IMF, the constant slope of the $N_{*}$ distribution extending to the field stars has profound consequences for their global feedback influence in galaxies. We can now quantify the assertion that most massive stars form in associations: for an $N_{*}$ distribution given by equa- tion 1 , the total number of OB stars is,

$$
N_{*, \text { tot }} \propto \sum_{N_{*}=1}^{N_{*, \text { up }}} N_{*} \cdot N_{*}^{-2},
$$

where $N_{* \text {,up }}$ refers to the upper-limit, maximum cluster of stars. Equation 9 corresponds to a divergent harmonic series which can be approximated for large $N_{*, \text { up }}$ as:

$$
N_{*, \text { tot }} \stackrel{\propto}{\sim} \ln N_{*, \text { up }}+\gamma
$$

where $\gamma \simeq 0.5772$ is the Euler-Mascheroni constant. Thus the fraction of $N_{*}=1$ field stars is $\left(\ln N_{* \text {,up }}+\gamma\right)^{-1}$ of the total $N_{* \text {,tot }}$. For our OB and O star samples, respectively, $\log N_{*, \text { up }} \simeq 2.0$ and 1.8, yielding $19 \%$ and $21 \%$ fractions for the field stars.

Counting the actual stars in our OB and O star samples, we find that 374 and 91 candidates, respectively, had no massive companions within the clustering radius. These correspond to $28 \%$ and $24 \%$, reflecting the excess found above. These fractions are about a factor two lower than the finding by Parker et al. (2001) that over half of their UIT-selected candidate O stars are outside catalogued association boundaries in the Large Magellanic Cloud (LMC). The results for both the SMC and LMC may be odds with the results of Massey et al. (2002), who found a much steeper IMF slope for field vs cluster massive stars: a steeper IMF slope in the field would be manifested as a smaller number of field OB stars, yet the slope of the $N_{*}$ distribution tends to be steeper than expected, rather than flatter. If the field star IMF is indeed steeper than in clusters, then the clustering law must also steepen substantially for the smallest clusters, in such a way as to compensate for a steep IMF in our data. Further investigation is necessary to resolve this issue. 

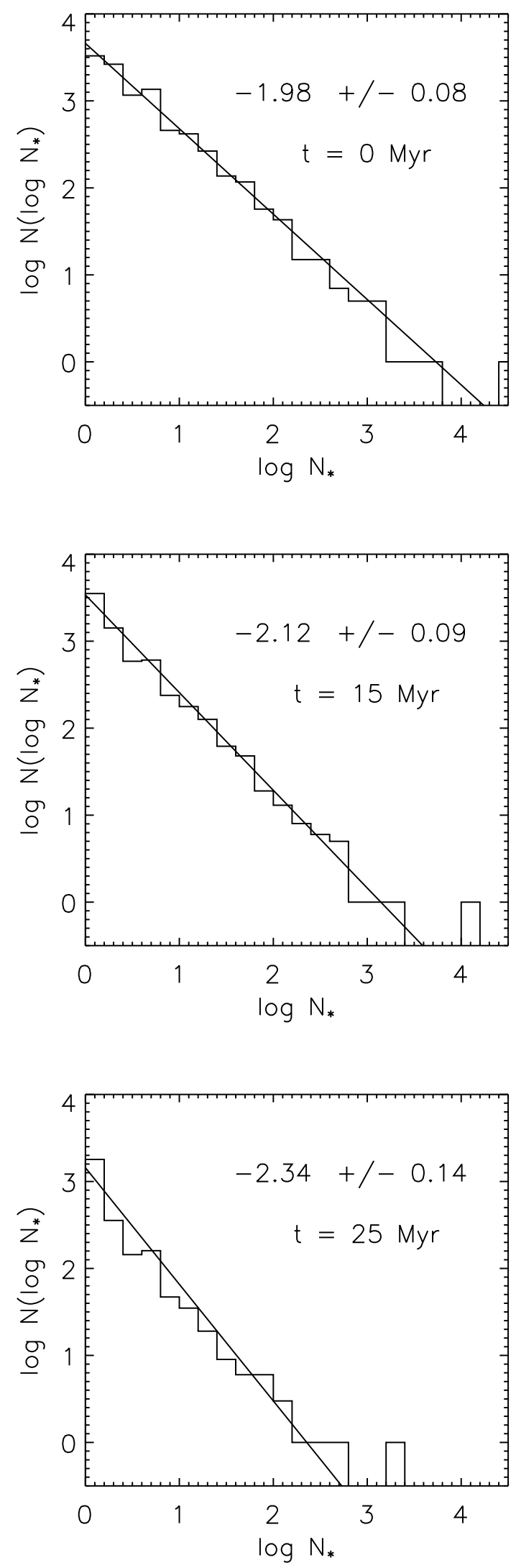

FIG. 8. - Monte Carlo models for evolution in the $\log N_{*}$ distribution, assuming that all objects were created simultaneously. Models for ages of 0,15 , and $25 \mathrm{Myr}$ are shown, with fitted power-law slopes. As in Figure 5, the linear power-law exponents are shown, while the fitted slope is $-\beta+1$ in logarithmic space. 


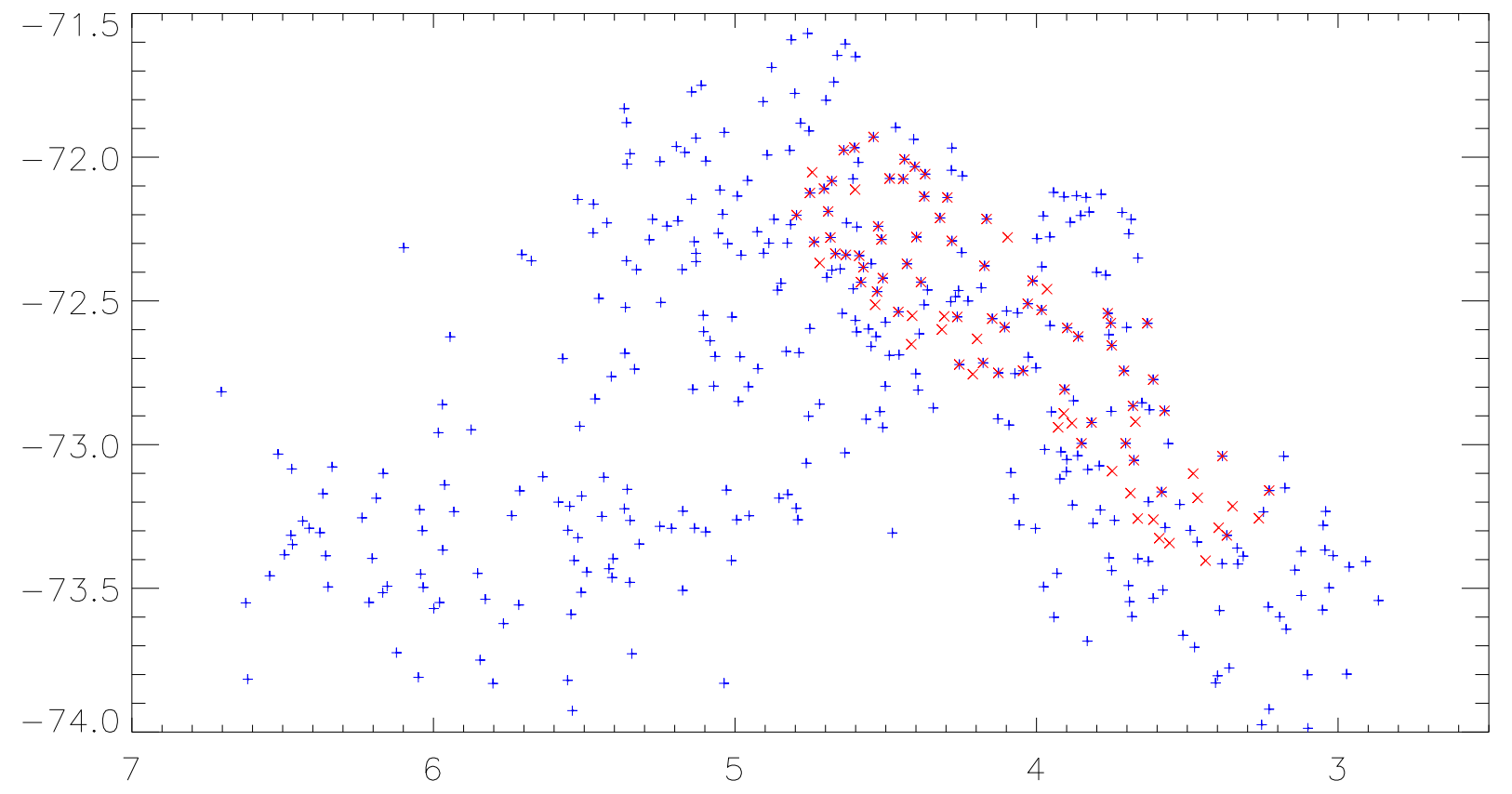

Fig. 9. - Spatial distribution of isolated field stars for the OB sample (plus symbols), and O-star sample (cross symbols). Scale and axes are as in Figure 3.

In the meantime, the data appear to be broadly consistent with the simpler scenario of, simultaneously, a universal IMF and universal clustering law given by equation 1. As emphasized by McKee \& Williams (1997), the total OB star population, and thus the fraction of isolated field massive stars, is driven by $N_{*}$,up with an inverse logarithmic dependence. For maximum $N_{* \text {,up }}$ ranging between 10 and $10^{6}$, equation 10 yields a fraction of field OB stars ranging from $35 \%$ to $7 \%$, respectively.

The clustering law in equation 1 has important consequences for feedback, implying that the interstellar porosity caused by the formation of superbubbles and supernova remnants has equal relative contributions from objects of all sizes (Oey \& Clarke 1997). And, since the Strömgren volume $V_{s} \propto N_{*}$, it also implies that a strong majority of the field massive stars likely contribute to ionizing the diffuse, warm ionized medium, which constitutes about $40 \%$ of the total $\mathrm{H} \alpha$ luminosity in star-forming galaxies (e.g., Walterbos 1998). Our result is quantitatively consistent with the result of Hoopes \& Walterbos (2000) that field OB stars can power $40 \% \pm 12 \%$ of the warm ionized medium in M33, where the fraction of field OB stars is likely around $15 \%$.

\section{CONCLUSION}

We find no evidence that the field massive stars in the SMC are formed by a fundamentally different star-forming process. Rather, we find that the continuous power-law distribution in $N_{*}$ down to $N_{*}=1$ strongly suggests that the star-forming process is continuous from rich clusters to poor groups, apparently for all ensembles that form $\mathrm{OB}$ stars. The data are consistent with the model that single, field $\mathrm{OB}$ stars are usually the most massive member of a group of smaller stars, as expected from the universal $N_{*}$ distribution (equation 1). These results are consistent with the simultaneous existence of a universal IMF and universal $N_{*}^{-2}$ clustering law. These joint universal power laws imply that field OB stars constitute roughly $35 \%$ to $7 \%$ of the total massive star population, with an inverse logarithmic dependence on $N_{*, \text { up }}$ of the the most populous cluster. Thus, the fraction is dependent on galaxy size and/or star formation rate. The contribution of these field stars to the ionized volume in the warm ionized medium is likely to scale according to their relative fraction. The universal clustering law also implies equal relative contributions by superbubbles of all sizes to the interstellar porosity.

We are pleased to acknowledge discussions with René Walterbos, Cristiano Porciani, Todd Small, and especially, Chris McKee and the anonymous referee. We also thank Phil Massey for generously providing the $U B V R$ data in advance of publication. This work was supported by the NASA Astrophysics Data Program, grant NAG5-10768.

\section{REFERENCES}

Azzopardi, M. \& Vigneau, J. 1982, A\&AS, 291

Battinelli, P. 1991, A\&A, 244, 69

Blaaw, A. 1961, Bull. Astron. Inst. Netherlands, 15, 265

Bothun, G. D. \& Thompson, I. B. 1988, AJ, 96, 87
Charbonnel, C.. Meynet, G., Maeder, A., Schaller, G., \& Schaerer, D. 1993, A\&A, 101, 415

Cardelli, J. A., Clayton, G. C., \& Mathis, J. S. 1989, ApJ, 345, 245

Dolphin, A. E., Walker, A. R., Hodge, P. W., Mateo, M., Olszewski, E. W., Schommer, R. A., \& Suntzeff, N. B. 2001, ApJ, 562, 303 
Elmegreen, B. G. \& Efremov, Y. N. 1997, ApJ, 480, 235

Feast, M. W., Thackeray, A. D., \& Wesselink, A. J. 1960, MNRAS, 121,337

Gies, D. R. 1987, ApJS, 64, 545

Harris, W. E. \& Pudritz, R. E 1994, ApJ, 429, 177

Hodge, P. W. 1985, PASP, 97, 530

Hoopes, C. G. \& Walterbos, R. A. M. 2000, ApJ, 541, 597

Hunter, D. A., Elmegreen, B. G., Dupuy, T. J., \& Mortonson, M. 2003, AJ, 126, 1836

Kennicutt R. C., Edgar B. K., \& Hodge P. W., 1989, ApJ, 337, 761

Kroupa, P. \& Weidner C. 2003, ApJ, in press; astro-ph/0308356

Landolt, A. U., 1992, AJ, 104, 340

Li, Y., Klessen, R. S., \& Mac Low, M.-M. 2003, ApJ, 592, 975

Lucke, P. B. \& Hodge, P. W. 1970, AJ, 75, 171

Massey, P., 2002, ApJS, 141, 81

McKee, C. F. \& Williams, J. P. 1997, ApJ, 476, 144
Meurer, G. R., Heckman, T. M., Leitherer, C., Kinney, A., Robert, C., \& Garnett, D. R. 1995, AJ, 110, 2665

Oey, M. S. \& Clarke, C. J. 1997, MNRAS, 289, 570

Oey, M. S. \& Clarke, C. J. 1998, AJ, 115, 1543

Oey, M. S. \& Clarke, C. J. 2003, in preparation

Oey, M. S. \& Muñoz-Tuñon, C. 2003, in Star Formation Through Time, eds. E. Pérez, R. González Delgado, \& G. Tenorio-Tagle, (San Francisco: ASP), 61

Parker, J. W., Cornett, R. H., \& Stecher, T. P. 2004, in preparation

Parker, J. W., Hill, J. K., Cornett, R. H., et al. 1998, AJ, 116, 180

Parker, J. W., Zaritsky, D., Stecher, T. P., Harris, J., \& Massey, P. 2001, ApJ, 121, 891

Salpeter E. E. 1955, ApJ, 121, 161

Sanduleak, N. 1969, Contr. Cerro-Tololo Interamerican Obs., 89

Staveley-Smith, L., Sault, R. J., Hatzidimitriou, D., Kesteven, M. J., \& McConnell, D. 1997, MNRAS, 289, 225

Zhang, Q. \& Fall, S. M. 1999, ApJ, 527, L81 